\title{
Long-term outcomes for 2-stage urethroplasty: an analysis of risk factors for urethral stricture recurrence
}

\author{
James R. Furr ${ }^{1}$ · Eric S. Wisenbaugh ${ }^{1}$ · Joel Gelman ${ }^{2}$
}

Received: 22 December 2020 / Accepted: 16 March 2021 / Published online: 3 April 2021

(c) The Author(s) 2021

\begin{abstract}
Purpose To report long-term results and patient reported outcomes of staged anterior urethroplasties, and isolate risk factors for recurrence.

Methods We reviewed urethroplasty database for all patients who underwent staged urethroplasty from 2000 to 2017. Follow-up included a cystoscopy 4 months after their 2 nd stage to assess early success, and then annual follow-up thereafter with post-void residual and symptom assessment. Stricture characteristics, etiology and graft type were analyzed with regards to success.

Results Forty-nine patients were eligible for inclusion. The median stricture length was $7 \mathrm{~cm}(3-17 \mathrm{~cm})$. The early success rate demonstrated by cystoscopy at 4 months was $100 \%$. Long-term success was $96.4 \%$ in buccal graft (BMG) only patients; however, long-term success fell considerably to 53\% in patients requiring any use split thickness skin graft (STSG) in the first stage. Median follow up time was 57 months (6-240 months). On analysis, age, increased stricture length and especially the use of STSG all appeared to be associated with late recurrence. The recurrence group had longer stricture length and were more likely to be panurethral. All recurrences occurred after the initial 4-month cystoscopy with a median time to recurrence of 78 months.

Conclusion Staged repairs that are amenable to BMG-only repairs have high long-term success rates. Increasing stricture length and the addition of split-thickness skin graft were associated with lower success rate in staged urethral reconstruction. Patients requiring staged repairs often experience recurrence in a very delayed fashion reinforcing the need for close, long-term follow up.
\end{abstract}

Keywords Urethral stricture $\cdot$ Urethroplasty $\cdot$ Staged urethroplasty $\cdot$ Split thickness skin graft $\cdot$ Buccal graft

\section{Introduction}

Staged urethroplasties are becoming less frequent as many long, complex urethral strictures are now being repaired in a single stage [1]. However, certain strictures are at times not amenable to single stage repairs due to a corpus spongiosum or urethral plate that is unsuitable for augmentation. As a

Joel Gelman

jgelman@uci.edu

James R. Furr

james-furr@ouhsc.edu

1 University of Oklahoma College of Medicine, 920 Stanton L. Young BLVD, WP 2140, Oklahoma City, OK 73104, USA

2 Department of Urology, University of California, 333 City Blvd West, Suite 1240, IrvineOrange, CA 92868, USA consequence, staged repairs are still commonly performed for strictures as a result of failed prior hypospadias surgery, highly complex strictures that have been treated with prior open reconstruction, and select complex cases of lichen sclerosus (LS) also known as Balanitis Xerotica Obliterans (BXO).

It is now clearly established that genital skin is not recommended for the treatment of urethral strictures associated with LS when used as flap or grafts [2]. Despite early success, this change in clinical practice away from the use of genital skin occured because of the very high late failure rate that was observed when there was long-term follow-up of up to 10 years [3,4]. The fact that substitution urethroplasty including staged repairs can in certain cases, be associated with a high late failure rate indicates the importance of longterm outcome assessment. 
Schrieter et al. first described the use of split thickness skin grafts (STSG) including extra-genital grafts for a 1st stage operation [5,6]. Buccal mucosa is now the graft material of choice for single-stage repairs due to excellent overall success rates, and is now being used with increased frequency for staged repairs $[1,7]$. However, the quantity of available oral mucosa is limited. Sufficient graft material must be harvested to insure tubularization to a normal caliber during the second stage repair along the entire area of stricture in complex, panurethral disease. One advantage of the use of STSG from the thigh is the ease and speed of harvesting sufficient graft material. While patients with stricture disease that requires a staged approach to urethroplasty are a relative minority, they often represent some of the most challenging patients in urethral reconstruction.

Although the use of extra-genital STSG and buccal mucosa grafts (BMG) are currently favored for staged urethroplasty, there are gaps in the current published literature. The reported short-term success rate is generally high. However, the limitations of the current literature include relatively short follow-up, the inclusion of multiple different surgical techniques without separate assessment of a stagedurethroplasty outcomes, or the lack of a clearly defined follow-up protocol to assess post-operative anatomic success and/or long-term results [8-10]. We aim to present an analysis of our series of staged urethroplasties using BMGs and/or STGT harvested from the thigh to include early assessment of anatomic success and long-term follow-up with patient reported outcome measures (PROMs). It was hypothesized that with longer follow-up, the late recurrence rate would increase, and that the inclusion of skin grafts could represent a risk factor for late failures.

\section{Materials and methods}

\section{Patient selection and preoperative evaluation}

With IRB approval, a prospectively maintained urethroplasty database was queried isolating all patients who underwent staged repair from 2000 to 2017. Pre-operatively, all patients underwent a flexible urethroscopy, a retrograde urethrogram, and a voiding cystourethrogram. Candidates for staged urethroplasty were selected in accordance with treatment algorithms previously described [11]. In short, patients who underwent staged repairs generally lacked an intact corpus spongiosum or urethral plate that could be adequately mobilized and augmented with a substitution 1-stage urethroplasty. As such, the study included hypospadias surgery failures, prior reconstructive failures, and certain patients with complex lichen sclerosus that extended into the fossa navicularis. Isolated meatal and fossa strictures were excluded. Additionally, patients were excluded if any portion of their repair was performed in a single stage, or if they elected to not pursue a second stage urethroplasty.

\section{Operative technique}

The 1st stage was performed by making a ventral urethrotomy through the stricture, ensuring that the urethra proximally was non-hirsute and widely patent (ideally $30 \mathrm{Fr}$ ). We accomplished this by bougie calibration and cystoscopy once the entire stricture had been incised. The urethral plate was typically left in the midline, although it was excised if it did not contribute significantly to urethral width or was hirsute. The goal was to transfer enough graft to allow the entire area of the recipient bed to measure slightly more than $3 \mathrm{~cm}$ in width, to allow for a 30Fr urethra when tubularized during the 2nd stage surgery. Early in our series, we performed staged repairs on two patients using STSG alone harvested from the thigh. As BMGs gained popularity, we began to favor the inclusion of buccal grafts along with or instead of STSG. However, with longer complex strictures that required extensive tissue transfer, STSG was used when bilateral buccal grafts did not provide sufficient graft material.

The 2nd stage, performed at least 4 months later, involved tubularization of the neo-urethra followed by a multi-layer closure. We attempt to bring the meatus as distally as possible, but did not risk sacrificing caliber to bring it to the most distal aspect of the glans. Pictures illustrating the different surgical steps are displayed in Fig. 1.

\section{Post-operative care}

A bolster was applied at the end of the 1st stage operation and kept in place for 5 days. The bolster consisted of xeroform gauze directly applied to the graft with overlying dacron wool soaked in mineral oil, all secured with sutures to skin; the urethral stenting catheter was removed approximately 10-14 days after surgery. Following bolster removal, patients were asked to keep xeroform gauze on the graft for another 14 days.

Patients were assessed 4 months after first-stage repair to confirm adequate graft for tubularization, wide patency of the ventrally displaced meatus, and a wide caliber of the urethra proximal to the meatus with flexible cystoscopy. After the 2nd stage procedure, the urethral stenting catheter was removed at 3 weeks, and a voiding cystourethrogram was obtained. Our follow-up protocol after the 2nd stage included distal urethral calibration with bougie-a-boules and cystoscopy at 4 months to ensure early success, and then annual follow-up thereafter with a post-void residual and symptom assessment to assess long-term success. At each annual follow-up, office staff collected patient reported outcomes such as the International Prostate Symptom Score 

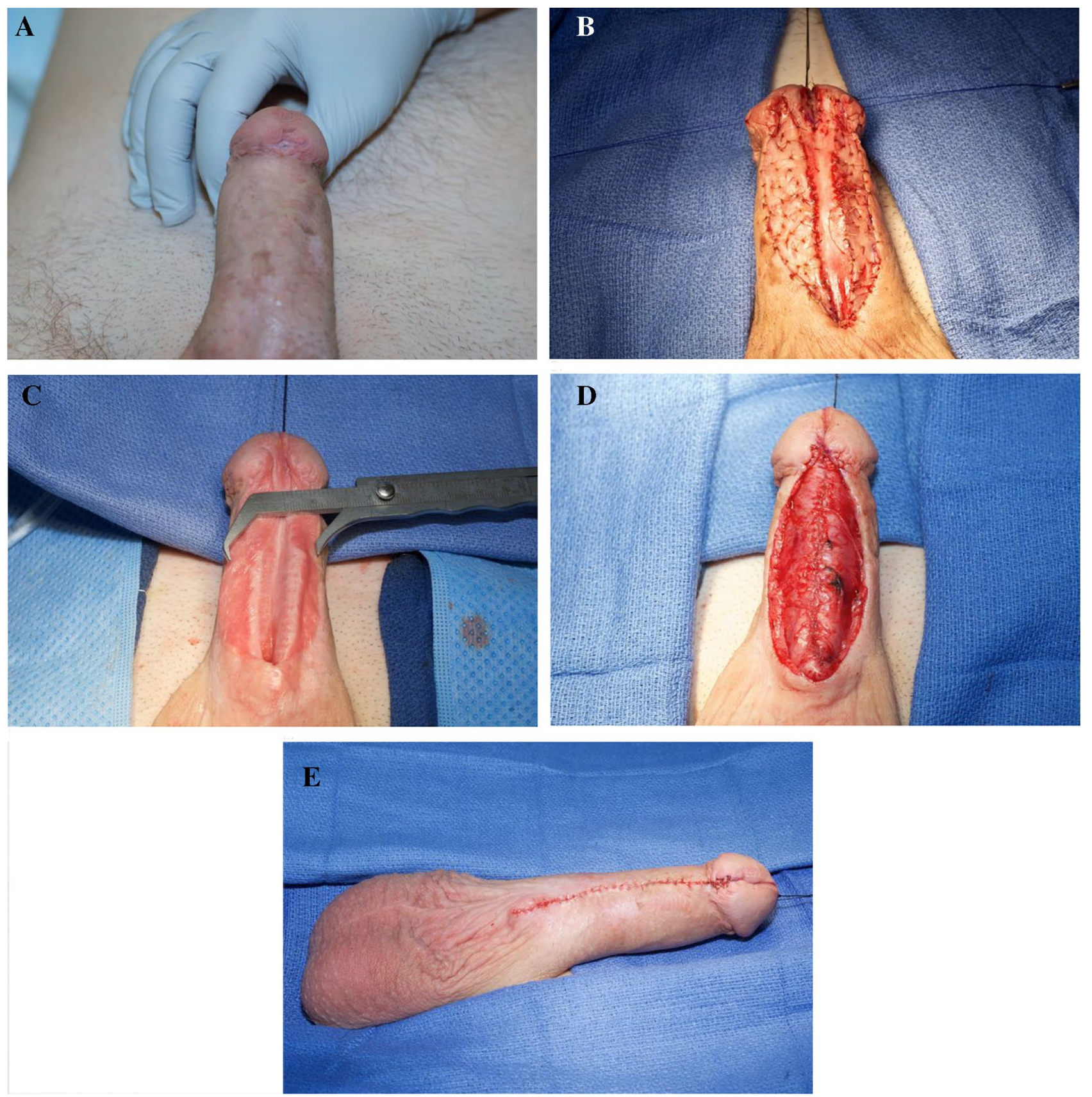

Fig. 1 a Patient with a long penile urethral stricture and history of hypospadias. b The ventral urethrotomy has been made and BMG fixated to either side of the urethral plate. c Demonstration of $3 \mathrm{~cm}$ width to the healed urethral plate to allow for a $30 \mathrm{Fr}$ caliber lumen. D\&E) Closure is accomplished in multiple layers

\section{Data analysis}

Patient characteristics such as age, stricture length, comorbid conditions such as LS or hypospadias, and the use of BMG and/or STSG were isolated The primary outcome was urethral patency, with a particular focus on the longterm success rate. Other endpoints assessed were patient reported outcomes (PROMS). Early anatomic success was 
Table 1 Patient Demographics and Outcomes of Staged Urethroplasties

\begin{tabular}{ll}
\hline Number of patients & 49 \\
Median age (years) & 37 (range 16-75) \\
Mean age (years) & $37.6(95 \%$ CI 33.2-42.0) \\
Diagnosis (\%) & \\
Hypospadias & $37(75.5 \%)$ \\
LS & $7(14.3 \%)$ \\
Both & $2(4.1 \%)$ \\
Neither/Recurrent Stricture Disease & $3(6.1 \%)$ \\
Location & \\
Meatal/Fossa extending to Penile & $27(55 \%)$ \\
Penobulbar/Panurethral & $22(45 \%)$ \\
Prior failed reconstruction (\%) & 39 (80\%) \\
Median stricture length (cm) & 7 (range 3-17) \\
Mean stricture length (cm) & $7.5(95 \%$ CI 6.3-8.6) \\
Median post op IPSS & 4 (range 0-13) \\
Median post op SHIM & 20 (range 0-25) \\
\% Worsening sex & $17.5 \%$ \\
Median follow-up & 57 months (range 6-250) \\
Mean follow-up & 63.7 months (95\% CI 54.2-83.6) \\
Median time between stages & 6 months (range: 4-75) \\
Median delay in starting stream (0 "never"— "all the time") & 0 \\
Median post void dribbling (0 "never"-4 "all the time") & 1 \\
Complications (\%) & $4(10 \%)$ \\
Satisfaction rate & $86 \%$ \\
\% Would have surgery again & $100 \%$ \\
Early technical success & $100 \%$ \\
\hline &
\end{tabular}

defined as the ability to easily pass a $16 \mathrm{Fr}$ cystoscope at the 4-month cystoscopy after the 2 nd stage repair (correlating to early, technical success). Long-term functional success was defined as either an absence of lower urinary tract symptoms based on history and IPSS and other urethroplasty-specific and satisfaction-related PROMs, or confirmation of continued wide urethral patency on repeat cystoscopy if there was development of obstructive voiding symptoms, elevated residuals or urinary tract infections. We did not have defined "triggers" such as a certain numerical change in a symptom score as an indication to perform a follow-up cystoscopy. The decision was often related a reported adverse change in symptoms such as a decreased force of stream, and/or a decrease in satisfaction due to an adverse voiding symptom change. Length of follow-up was determined by the interval between the date of surgery and the date of most recent evaluation, not the time between the date of surgery and manuscript submission. Both mean and median based data were incorporated into our analysis for continuous variables, specifically age, stricture length and follow-up. Non-parametric mann-whitney $U$, chi-squared, and Fisher's exact tests (for categorical data) were used when appropriate, and a $t$-test was also included in analysis for continuous, mean-based variables. Analysis was performed with StataSE (College Station, TX). Statistical significance was set at $p<0.05$.

\section{Results}

A total of 85 patients underwent 1st stage repairs during the study period, of which 57 patients underwent both 1st and 2 nd stage repairs. No patient required a revision surgery subsequent to the 1 st stage repair to add additional graft to insure sufficient width $(30+\mathrm{mm})$ for tubularization. One young patient who had a pan-urethral stricture after failed proximal hypospadias repairs and underwent 1st stage repair with STSG and BMG did undergo a procedure to add buccal mucosa to the fossa navicularis prior to the second stage repair. The objective was to widen the fossa with a mucosal graft that would provide a more normal appearing urethral opening after tubularization.

In total, 49 were compliant with follow-up and eligible for inclusion. Two of these 49 patients who were compliant with long-term follow-up did not return for their 4-month cystoscopy, and they were included only in the long-term functional outcome analysis. There were 5 patients who underwent cystoscopy at 4 months, but did not have 12 or 
Table 2 Comparative Analysis of Outcomes

\begin{tabular}{|c|c|c|c|c|c|c|}
\hline & \multicolumn{3}{|c|}{ Analysis of recurrence } & \multicolumn{3}{|c|}{ Comparison of BMG vs. STSG outcomes } \\
\hline & Success $(n=40)$ & Recurrence $(n=9)$ & $P$-values & $\begin{array}{l}\text { STSG in } \\
\text { any portion } \\
(n=17)\end{array}$ & BMG only $(n=32)$ & $P$-values \\
\hline Median Age (years) & 34 & 48 & $\mathbf{0 . 0 2}$ (Mann-Whitney $U$ ) & 37 & 35 & 0.93 \\
\hline Mean Age (years) & 35.3 & 47.9 & $\mathbf{0 . 0 2}$ (T-test) & 38.2 & 37.4 & 0.93 \\
\hline Etiology $(n=)$ & & & 0.515 & & & 0.32 \\
\hline$\%$ Hypospadias & $77.5 \%$ & $66.7 \%$ & & $64.7 \%$ & $81.25 \%$ & \\
\hline$\% \mathrm{LS}$ & $17.5 \%$ & $22.2 \%$ & & $29.4 \%$ & $12.5 \%$ & \\
\hline $\begin{array}{l}\text { \% Only Multiple Failed } \\
\text { Reconstruction }\end{array}$ & $5.0 \%$ & $11.1 \%$ & & $5.9 \%$ & $6.25 \%$ & \\
\hline $\begin{array}{l}\text { Median Stricture Length } \\
(\mathrm{cm})\end{array}$ & 5.0 & 8.0 & 0.05 (Mann-Whitney $U$ ) & 10 & 5 & $<0.001$ \\
\hline Mean Stricture Length $(\mathrm{cm})$ & 6.8 & 10.0 & $\mathbf{0 . 0 2}$ ( $T$-test $)$ & 10.2 & 5.9 & 0.003 \\
\hline Location & & & 0.12 & & & $<0.001$ \\
\hline $\begin{array}{l}\% \text { Meatal/Fossa extending } \\
\text { to Penile }(n=)\end{array}$ & $60.0 \%(24)$ & $33.3 \%(3)$ & & $19 \%(3)$ & $75 \%(24)$ & \\
\hline $\begin{array}{l}\text { \%Bulbopenile/Panurethral } \\
(n=)\end{array}$ & $40.0 \%(16)$ & $66.7 \%(6)$ & & $81 \%(14)$ & $25 \%(8)$ & \\
\hline Median Post Op IPSS & 3 & 5 & 0.25 & 4 & 3 & 0.56 \\
\hline $\begin{array}{l}\text { Median Delay in Starting } \\
\text { Stream (0 "never" }-4 \text { "all } \\
\text { the time") }\end{array}$ & 0 & 2 & 0.18 & 0 & 1 & 0.35 \\
\hline $\begin{array}{l}\text { Median Post Void Dribbling } \\
\text { (0 "never" }-4 \text { "all the time") }\end{array}$ & 1 & 1 & 0.26 & 1 & 1 & 0.94 \\
\hline Median Post Op SHIM & 20 & 15 & 0.55 & 13 & 15 & 0.69 \\
\hline$\%$ Worsening Sex $(n=)$ & $17.2 \%(6)$ & $20.0 \%$ & 0.64 & $17.8 \%(5)$ & $16.7 \%(2)$ & 0.654 \\
\hline$\%$ with STSG $(n=)$ & $22.5 \%(9)$ & $88.9 \%(9)$ & $<0.001$ & & & \\
\hline $\begin{array}{l}\text { Long-term Success } \\
\quad(>1 \text { year })^{\mathrm{a}}\end{array}$ & & & & $53 \%$ & $96.4 \%$ & $<0.001$ \\
\hline $\begin{array}{l}\text { Median Length of Follow-up } \\
\text { (months) }\end{array}$ & 46.03 & 127.9 & 0.001 (Mann-Whitney $U$ ) & 79 & 45 & 0.02 \\
\hline $\begin{array}{l}\text { Mean length of follow-up } \\
\text { (months) }\end{array}$ & 55.8 & 127.9 & $<\mathbf{0 . 0 0 1}(T$-test $)$ & 94.9 & 55.5 & 0.027 \\
\hline Satisfaction & $86 \%$ & $50 \%$ & 0.06 & $73 \%$ & $86 \%$ & 0.275 \\
\hline
\end{tabular}

Bold values signify outcomes that are statistically significant

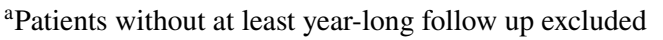

Table 3 Summary of recurrences

\begin{tabular}{llllllll}
\hline & Graft & Etiology & Age (Years) & $\begin{array}{l}\text { Recurrence } \\
\text { time (Month) }\end{array}$ & $\begin{array}{l}\text { Stricture } \\
\text { length }(\mathrm{cm}) *\end{array}$ & Complications & Management \\
\hline 1 & STSG only & LS & 54 & 22 & 17 & Extended meatotomy \\
2 & STSG only & Failed flap & 33 & 96 & 7 & Fistula & Repeat buccal urethroplasty \\
3 & Buccal-STSG & Hypospadias & 48 & 162 & 6 & Perineal Urethrostomy \\
4 & Buccal-STSG & Hypospadias & 44 & 120 & 15 & Perineal Urethrostomy \\
5 & Buccal-STSG & Hypospadias & 54 & 78 & 8 & Repeat urethroplasty \\
6 & Buccal-STSG & Hypospadias & 63 & 26 & 15 & DVIU with self-dilations \\
7 & Buccal-STSG & Hypospadias & 49 & 29 & 6 & Observation \\
8 & Buccal-STSG & Hypospadias & 69 & 24 & 11 & Perineal Urethrostomy \\
9 & Buccal & LS & 27 & 155 & 4.5 & Extended meatotomy \\
\hline
\end{tabular}

*Stricture length refers to original stricture length 
more months of follow-up after surgery. They were excluded from the long-term analysis.

Of included patients, 37 (77\%) had a diagnosis of hypospadias, 7 (15\%) had LS, 2 had both, and three had very complex, recurrent strictures in the absence of either diagnosis. The vast majority (80\%) had a history of prior failed urethroplasty. The median stricture length was $7 \mathrm{~cm}$ (range 3-17 cm) with a median follow-up of 57 months (range 6-240 months). Median time between first and second stages was 6 months. Patient demographics for the staged urethroplasty cohort are summarized in Table 1 .

STSG and BMG was used in the first stage in 15 patients, with 2 patients undergoing STSG-only first stages early on in our series. This is compared to 32 patients whose 1 st stage was grafted with BMG only. The STSG group trended toward longer median stricture length $(8 \mathrm{~cm}$ vs $5 \mathrm{~cm})$. However, no differences were noted in rate of LS or hypospadias in each group as can be seen in Table 2 .

The early technical success rate for all patients in both groups demonstrated by cystoscopy at 4 months following $2^{\text {nd }}$ stage reconstruction was $100 \%$ (Table 1 ). We also noted a $96.4 \%$ long-term success in BMG only patients with one late recurrence isolated to the distal $\mathrm{cm}$ of the urethra; however, long-term success fell considerably to $53 \%$ in patients requiring any use STSG in the first stage, which included late recurrences in both of the men whose firststage consisted only of STSG. Only patients with follow-up time of $>1$ year were included in the analysis of long-term follow-up, with four patients being excluded. Mean time to recurrence was 95 months, and median time was 78 months (range 22-164 months). Complications (outside of stricture recurrence) were experienced in $10 \%(n=5)$, which included two urethrocutaneous fistulas, one patient with significant meatal redundancy of buccal graft after second stage requiring revision, and two urinary tract infections. No patient had a donor site complication.

On univariate analysis, age, the use of STSG and increased stricture length all appeared to be associated with repair failure. The recurrence group trended towards significantly longer length and were more likely to be panurethral (Table 2). With regards to questionnaire data and PROMs, the cohort as a whole had favorable post op IPSS and SHIM results (Table 1). Additionally, the rates of post void dribbling and delay in starting stream was low, with median answers of $<1$, correlating between "never" and "occasionally" on the questioniarre. While not statistically significant, these outcomes tended to be worse in patients who had STSG and who had recurrences (Table 2). Overall satisfaction rate was $86 \%$, and $100 \%$ of patients stated they would have the surgery again. However, four patients who recurred declined to answer the satisfaction portion of the PROM questionnaire, and could likely be assumed to be unsatisfied. Of the patients that were unsatified, three stated that their symptoms did not improve, one patient was bothered by post void dribbling. Another complained of recurrent infections despite anatomic success, and one last patient had bother associated with decreased sensation. Of the nine recurrences, eight underwent retreatment, and one continued observation (Table 3).

\section{Discussion}

\section{Definitions of early and long-term urethroplasty success}

While it is ideal to repair a urethral stricture with one operation, those with highly complex strictures following multiple repairs, prior hypospadias or LS often are often best suited to a staged approach. The reported success of staged repairs has varied considerably in published reports, and a major factor in this wide variation may be the different definitions of success along with differences in the length of followup [13]. Anatomic urethroplasty success is often defined as wide patency confirmed with cystoscopy 4-12 months after surgery [14]. This is a reflection of early and technical success. Another outcome measure is functional success that can be defined as an absence of symptoms, peak flow rates of at least $12-15 \mathrm{ml} / \mathrm{s}$, or if there is no need for future stricture treatment. There is the potential for these definitions of success to underestimate recurrences. For example, it is quite possible that a man with a symptomatic recurrent stricture did not elect to pursue further treatment. In addition, it has been shown that the use of International Prostate Symptom Score (IPSS) alone is an inadequate tool to screen for stricture recurrence [15]. Moreover, changes in peak flow rate may be associated with recurrent strictures rather than wide urethral patency [16]. The most appropriate definition of a successful urethroplasty should incorporate both objective (anatomic) and subjective (functional) outcome measures to include specific patient reported outcome measures (PROMs), including quality of life and satisfaction assessment, and all of these definitions were incorporated into our study protocol [17].

Although a particular urethroplasty can associated with both early anatomic and early functional success using the above definitions, the durability of an initially successful repair is of major importance when assessing the utility and value of a substitution technique. In contrast to urethral reconstruction with excision and primary anastomosis, substitution urethroplasty can be especially prone to late recurrences after 5-10 + years [18]. A striking example of a surgery that is associated with good early success, but a very high late failure rate, is substitution urethroplasty using genital skin for the treatment of LS strictures. The use of genital skin as flaps or grafts in 1 or staged repair 
was once commonly performed, and was associated with good early success. However, when it was reported that with up to 10 years of follow-up, the recurrence rate approached $100 \%$, this technique was abandoned $[3,4,19]$. In contrast, comparisons supporting BMG use over other extra-genital graft material in staged repairs is scant.

\section{The use of staged repairs for urethroplasty}

The first description of the use of staged repairs using mesh foreskin and STSG grafts was by Schreiter [5, 6]. The reported early success rate was $99 \%$ in 96 patients with the majority of patients having at least 2 years of follow-up [6]. This technique was subsequently adopted by others. In 1997, Webster's group reported an $80 \%$ success rate in 20 men with a median follow-up of 38 months using STSG from the thigh in the vast majority of the cases [20]. This was followed by reports of the use of extra-genital grafts using buccal mucosa and postauricular skin to treat LS strictures with high success rate of $94 \%$ at a mean follow-up of 3 years. None of the above studies from the older literature included a protocol to assess urethral patency using anatomic or functional PROMs.

When 2-stage buccal mucosa urethroplasty was initially reported, the success rate was $93 \%$ with a median follow-up of 18 months [21]. As buccal mucosa became more favored as a graft material for substitution urethroplasty, numerous reconstructive Urologists began to report their results with buccal grafts. In a multi-institutional evaluation of all treatments for long-segment strictures by Warner et. al, the patients who were treated with skin grafts as opposed to BMGs had a higher recurrence rate [22]. A limitation of this comparison was a mean follow-up of only 20 months, and it was not stated if the skin grafts were genital or extra-genital. More recently, multiple studies have compared the performance of STSG with BMG directly with regards to staged repairs. These reports have shown comparable success rates between STSG and BMG groups [23-25]. However, two studies by Pfalzgraf et al. and Kluth et al. are limited by follow-ups of less than 1 year [24, 25]. In a another study by Pfalzgraf et al., follow-up approached 3 years with comparable quality of life measures, though overall patient satisfaction was lower in the STSG group versus the BMG group (83\% versus $93.7 \%)$ [23].

Our results also indicate a very high early success rate defined by anatomic confirmation of wide patency of the repair 4 months after the 2 nd stage repair using cystoscopy. We had no early recurrences of stricture in any patient. Moreover, our long-term functional outcome analysis revealed durable results when buccal mucosa was the only graft material used with only one late distal recurrence. However, in contrast to our high success rate for staged repairs using BMGs, the late recurrence rate was only 53\% when any STSG was included in the repair. Our data determined that, in addition to the use of any STSG, increased stricture length, and to a minor extent, age were associated with recurrence. It is unclear if age is in and of itself is a risk factor for failure, but given the relatively low number of failures, subset analysis with a focus on age cannot be performed. Of the nine patients that had late recurrences, only two had a diagnosis of LS, suggesting that graft material and perhaps stricture length may represent more significant risk factors than stricture etiology. However, in some patients who presented with a history of multiple failed surgeries for stricture related or not related to hypospadias repair, the scarring and recurrence may have been related to associated LS. We did not routinely biopsy patient who underwent staged repair.

\section{Stricture length and the use of STSG as risk factors for late recurrence}

Stricture length has been implicated in contemporary studies as an independent risk factor for repair failure for singlestage urethroplasties [26, 27]. These results also confirm those data reported by Selim et al., who also found length to be a risk factor for failure in staged repairs [28]. Kozinn et al. also reported a large series of staged urethroplasties (all with BMG-only) and reported first stage failures with mean stricture length of $11.7 \mathrm{~cm}$, and second stage failures with mean stricture length of $14 \mathrm{~cm} \mathrm{[29].} \mathrm{Based} \mathrm{on} \mathrm{such} \mathrm{find-}$ ings, there appears to be a clear association with increased stricture length and failure.

The most striking finding was the major decline in long-term success rates when extra-genital STSG harvested from the thigh was used exclusively or included as a tissue transfer graft material compared to staged repairs where BMG alone was used. However, we want to stress the limitations of our study. The patients who underwent repair that included STSG tended to have longer and more complex strictures which could represent a risk factor for failure. However, although four of the patients who had recurrences after having both STSG and BMG placed had $>10 \mathrm{~cm}$ strictures, the other, the other four had relatively short $(4.5-8 \mathrm{~cm})$ strictures, which could have likely been treated with only BMG. We were less aggressive in using buccal mucosa alone earlier in our series. Therefore, late recurrences in these patients whose repairs included the use of STSG were not limited to only patients who had very long strictures. This is a qualitative observation as opposed to a quantitative analysis, and further research should be dedicated to determining what extent graft material plays a role in the durability of repairs for long, complex strictures.

In addition, the length of follow-up in the group that had repairs that included the use of STSG was considerably 
longer. In part, this was due to a shift towards a more aggressive use of oral mucosa later in our series, but certainly a longer follow-up is often associated with a higher late recurrence rate. Our failures all occurred late, with a median time to recurrence of 78 months (Table 4). This highlights the importance of the long-term follow-up, as prior studies with significantly shorter follow-up would not have captured any of these late recurrences. Therefore, although the mean follow-up in our BMG only patients was a mean of 55 months (Range 6-140 months), with longer follow-up, it is possible that we will see late recurrences in this group.

Given that repairs requiring BMG only had a 93\% longterm success rate with a drop off to $53 \%$ when STSG was added, despite the limitation of our study, this observation indicates that STSG may be an inferior graft material for staged urethroplasties. This finding also appears consistent with data previously suggested by Bracka et al. [6] and Warner et al. [22].

If the goal is to avoid the use of not only genital but also extra genital skin for very long narrow caliber pan-urethral strictures, there is the issue of limited graft material that can be obtained from bilateral buccal graft harvests. Other types of oral mucosa such as lower lip and lingual grafts have been harvested and used in single-stage procedures with good success and low complication rates [30-32]. These grafts have very similar characteristics to BMG and could possibly offer higher success rates if used in lieu of STSGs, but the morbidity of harvesting bilateral BMGs and lingual and/or lower lip grafts has not been well studied. Given our findings, we intend to avoid STSG in the future, and will harvest lower lip grafts in addition to bilateral buccal grafts when additional graft material is needed.

A novel aspect of our presented data, in addition to the long-term follow-up, is the inclusion of PROMs with validated questionnaires in patients undergoing staged urethroplasty. Pre operative data were not routinely collected early on in the series with limited pre-operative and post-operative comparisons. However, our data do show that successful staged repairs result in very adequate post-operative IPSS, low complication, and high satisfaction rates. These results compare well to that which is reported for successful onestage substitution repairs and excision and primary anastomosis $[33,34]$.

\section{Conclusion}

Staged repairs that are amenable to BMG-only repairs have high long-term success rates. Increasing stricture length and the use of split-thickness skin graft resulted in lower success rate in staged urethral reconstruction. These patients tended to have longer, complex strictures, but perhaps most importantly, recurrences typically occur in a delayed fashion reinforcing the need for close, long-term follow-up.

Author contributions JRF: data collection, data analysis, manuscript writing. EW: data collection, data analysis. JG: project development, manuscript writing.

\section{Declarations}

\section{Conflicts of Interest None.}

Research involving human participants and informed consent This study was approved by internal institutional review board. All patients included provided written informed consent.

Open Access This article is licensed under a Creative Commons Attribution 4.0 International License, which permits use, sharing, adaptation, distribution and reproduction in any medium or format, as long as you give appropriate credit to the original author(s) and the source, provide a link to the Creative Commons licence, and indicate if changes were made. The images or other third party material in this article are included in the article's Creative Commons licence, unless indicated otherwise in a credit line to the material. If material is not included in the article's Creative Commons licence and your intended use is not permitted by statutory regulation or exceeds the permitted use, you will need to obtain permission directly from the copyright holder. To view a copy of this licence, visit http://creativecommons.org/licenses/by/4.0/.

\section{References}

1. Kulkarni SB, Joshi PM, Venkatesan K (2012) Management of panurethral stricture disease in India. J Urol 188(3):824-830. https://doi.org/10.1016/j.juro.2012.05.020

2. Wessells H, Angermeier KW, Elliott S, Gonzalez CM, Kodama R, Peterson AC, Reston J, Rourke K, Stoffel JT, Vanni AJ, Voelzke BB, Zhao L, Santucci RA (2017) Male urethral stricture: American Urological Association Guideline. J Urol 197(1):182-190. https://doi.org/10.1016/j.juro.2016.07.087

3. Depasquale I, Park AJ, Bracka A (2000) The treatment of balanitis xerotica obliterans. BJU Int. 86(4):459-465. https://doi.org/10. 1046/j.1464-410x.2000.00772.x

4. Trivedi S, Kumar A, Goyal NK, Dwivedi US, Singh PB (2008) (2008) Urethral reconstruction in balanitis xerotica obliterans. Urol Int 81(3):285-289. https://doi.org/10.1159/000151405

5. Schrieter F (1984) Mesh-Graft urethroplasty: our experience with a new procedure. Eur Urol 10:338-344

6. Schreiter F, Noll F (1989) Mesh graft urethroplasty using split thickness skin graft or foreskin. J Urol 142(5):1223-1226

7. Blaschko SD, Harris CR, Zaid UB, Gaither T, Chu C, Alwaal A, McAninch JW, McCulloch CE, Breyer BN (2015) Trends, utilization, and immediate perioperative complications of urethroplasty in the United States: data from the national inpatient sample 2000-2010. Urology 85(5):1190-1194. https://doi.org/10.1016/j. urology.2015.01.008

8. Patel CK, Buckley JC, Zinman LN, Vanni AJ (2016) Outcomes for management of lichen sclerosus urethral strictures by 3 different techniques. Urology 91:215-221

9. Barbagli G, De Angelis M, Palminteri E, Lazzeri M (2006) Failed hypospadias repair presenting in adults. Eur Urol 49(5):887-894. https://doi.org/10.1016/j.eururo.2006.01.027 
10. Dubey D, Sehgal A, Srivastava A, Mandhani A, Kapoor R, Kumar A (2005) Buccal mucosal urethroplasty for balanitis xerotica obliterans related urethral strictures: the outcome of 1 and 2-stage techniques. J Urol. 173(2):463-466

11. Wisenbaugh ES, Gelman J (2015) The use of flaps and grafts in the treatment of urethral stricture disease. Adv Urol. https://doi. org/10.1155/2015/979868

12. Jackson MJ, Sciberras J, Mangera A, Brett A, Watkin N, N'dow JM, Chapple CR, Andrich DE, Pickard RS, Mundy AR (2011) Defining a patient-reported outcome measure for urethral stricture surgery. Eur Urol. 60(1):60-68. https://doi.org/10.1016/j.eururo. 2011.03.003 (Epub 2011 Mar 17. PMID: 21419566)

13. Mori RL, Angermeier KW (2015) Staged urethroplasty in the management of complex anterior urethral stricture disease. Transl Androl Urol 4(1):29-34. https://doi.org/10.3978/j.issn.2223-4683. 2015.01.10

14. Baradaran N, Fergus KB, Moses RA, Patel DP, Gaither TW, Voelzke BB, Smith TG 3rd, Erickson BA, Elliott SP, Alsikafi NF, Vanni AJ, Buckley J, Zhao LC, Myers JB, Breyer BN (2019) Clinical significance of cystoscopic urethral stricture recurrence after anterior urethroplasty: a multi-institution analysis from Trauma and Urologic Reconstructive Network of Surgeons (TURNS). World J Urol. 37(12):2763-2768. https://doi.org/10. 1007/s00345-019-02653-6 (Epub 2019 Feb 2. PMID: 30712091)

15. Tam CA, Elliott SP, Voelzke BB, Myers JB, Vanni AJ, Breyer BN, Smith TG 3rd, McClung CD, Erickson BA, Trauma and Urologic Reconstruction Network of Surgeons (TURNS) (2016) The International Prostate Symptom Score (IPSS) is an inadequate tool to screen for urethral stricture recurrence after anterior urethroplasty. Urology 95:197-201. https://doi.org/10.1016/j.urology.2016.04. 006 (PMID: 27109599; PMCID: PMC5002376)

16. Erickson BA, Breyer BN, McAninch JW (2011) Changes in uroflowmetry maximum flow rates after urethral reconstructive surgery as a means to predict for stricture recurrence. J Urol. 186(5):1934-1937. https://doi.org/10.1016/j.juro.2011.07.010 (Epub 2011 Sep 23. PMID: 21944128; PMCID: PMC3565613)

17. Erickson BA, Ghareeb GM (2019) Definition of successful treatment and optimal follow-up after urethral reconstruction for urethral stricture disease. Urol Clin N Am 44(1):1-9. https://doi.org/ 10.1016/j.ucl.2016.08.001 (PMID: 27908363)

18. Andrich DE, Dunglison N, Greenwell TJ, Mundy AR (2003) The long-term results of urethroplasty. J Urol. 170(1):90-92. https:// doi.org/10.1097/01.ju.0000069820.81726.00 (PMID: 12796652)

19. Venn SN, Mundy AR (1998) Urethroplasty for balanitis xerotica obliterans. Br J Urol. 81(5):735-737. https://doi.org/10.1046/j. 1464-410x.1998.00634.x (PMID: 9634051)

20. Carr LK, MacDiarmid SA, Webster GD (1997) Treatment of complex anterior urethral stricture disease with mesh graft urethroplasty. J Urol. 157(1):104-108 (PMID: 8976227)

21. Palminteri E, Lazzeri M, Guazzoni G, Turini D, Barbagli G (2002) New 2-stage buccal mucosal graft urethroplasty. J Urol. 167(1):130-132 (PMID: 11743290)

22. Warner JN, Malkawi I, Dhradkeh M, Joshi PM, Kulkarni SB, Lazzeri M, Barbagli G, Mori R, Angermeier KW, Storme O, Campos R, Velarde L, Gomez RG, Han JS, Gonzalez CM, Martinho D, Sandul A, Martins FE, Santucci RA (2015) A multi-institutional evaluation of the management and outcomes of long-segment urethral strictures. Urology 85(6):1483-1487. https://doi.org/10. 1016/j.urology.2015.01.041

23. Pfalzgraf D, Kluth L, Reiss P, Fisch M, Dahlem R (2015) Staged urethroplasty: comparison of early functional results and quality of life in mesh graft and buccal mucosa technique. Can J Urol 22(2):7720-7726

24. Pfalzgraf D, Olianas R, Schreiter F, Fisch M (2010) Two-staged urethroplasty: buccal mucosa and mesh graft techniques. Aktuelle Urol 41(Suppl 1):S5-9. https://doi.org/10.1055/s-0029-1224664

25. Kluth LA, Dahlem R, Reiss P, Pfalzgraf D, Becker A, Engel O, Chun FK, Fisch M, Ahyai SA (2013) Short-term outcome and morbidity of different contemporary urethroplasty techniques-a preliminary comparison. J Endourol 27(7):925-929. https://doi. org/10.1089/end.2013.0029

26. Breyer BN, McAninch JW, Whitson JM, Eisenberg ML, Mehdizadeh JF, Myers JB, Voelzke BB (2010) Multivariate analysis of risk factors for long-term urethroplasty outcome. J Urol 183(2):613-617. https://doi.org/10.1016/j.juro.2009.10.018

27. Chapman D, Kinnaird A, Rourke K (2017) Independent predictors of stricture recurrence following urethroplasty for isolated bulbar urethral strictures. J Urol 198(5):1107-1112. https://doi.org/10. 1016/j.juro.2017.05.006

28. Selim M, Salem S, Elsherif E, Badawy A, Elshazely M, Gawish M (2019) Outcome of staged buccal mucosal graft for repair of long segment anterior urethral stricture. BMC Urol 19(1):38. https:// doi.org/10.1186/s12894-019-0466-4

29. Kozinn SI, Harty NJ, Zinman L, Buckley JC (2013) Management of complex anterior urethral strictures with multistage buccal mucosa graft reconstruction. Urology 82(3):718-722. https://doi. org/10.1016/j.urology.2013.03.081

30. Kamp S, Knoll T, Osman M, Hacker A, Michel MS, Alken P (2005) Donor-site morbidity in buccal mucosa urethroplasty: lower lip or inner cheek? BJU Int 96(4):619-623. https://doi.org/ 10.1111/j.1464-410X.2005.05695.x

31. Kumar A, Goyal NK, Das SK, Trivedi S, Dwivedi US, Singh PB (2007) Oral complications after lingual mucosal graft harvest for urethroplasty. ANZ J Surg 77(11):970-973. https://doi.org/10. 1111/j.1445-2197.2007.04292.x

32. Simonato A, Gregori A, Ambruosi C, Venzano F, Varca V, Romagnoli A, Carmignani G (2008) Lingual mucosal graft urethroplasty for anterior urethral reconstruction. Eur Urol 54(1):79-85

33. Morey AF, McAninch JW, Duckett CP, Rogers RS (1998) American Urological Association symptom index in the assessment of urethroplasty outcomes. J Urol 159(4):1192-1194

34. Furr JR, Wisenbaugh ES, Gelman J (2019) Urinary and sexual outcomes following bulbar urethroplasty-an analysis of two common approaches. Urology. https://doi.org/10.1016/j.urology. 2019.02.042

Publisher's Note Springer Nature remains neutral with regard to jurisdictional claims in published maps and institutional affiliations. 